\title{
Growing Teratoma Syndrome, A Rare Case
}

\author{
Hasanuddin ${ }^{1 *}$ and Hafis Novyan ${ }^{2}$ \\ ${ }^{1}$ Gynecologic Oncology Consultant, Department of Obstetrics and Gynecology, Faculty of Medicine, Syiah Kuala University / Dr. \\ Zainoel Abidin General Hospital, Indonesia
}

${ }^{2}$ Obstetrics and Gynecology Resident, Faculty of Medicine, Syiah Kuala University / Dr. Zainoel Abidin General Hospital, Indonesia

*Corresponding author: Hasanuddin, Gynecologic Oncology Consultant, Department of Obstetrics and Gynecology, Faculty of Medicine, Syiah Kuala University / Dr. Zainoel Abidin General Hospital, Banda Aceh, Indonesia

ARTICLE INFO

Received: 凿 January 21, 2022

Published: February 01, 2022

Citation: Hasanuddin, Hafis Novyan. Growing Teratoma Syndrome, A Rare Case. Biomed J Sci \& Tech Res 41(3)2022. BJSTR. MS.ID.006615.

Keywords: Immature Teratoma; Growing Teratoma Syndrome; Second Look Laparotomy

\begin{abstract}
Introduction: Germ cell is ovarian malignancy that occurs in a woman, especially at the young age women. Immature teratoma is the second most common malignancy in cases of germ cell. Immature teratoma has a good cure rate after proper treatment, namely surgery and chemotherapy. Growing teratoma syndrome (GTS) is a rare condition among patients with immature teratoma which is characterized by enlargement or appearance of a new mass after adequate systemic chemotherapy, normal tumor serum markers, and mass histopathology results show the appearance of mature teratoma in the new mass.
\end{abstract}

Case Illustration: It is reported that an 18-year-old, unmarried woman came to the gynecological oncology polyclinic for routine control with complaints of abdominal pain and a palpable lump in the lower abdomen. A history of undergoing unilateral salpingo-oophorectomy surgery in 2019, with histopathological results of immature teratoma and underwent completed chemotherapy with Bleomycin, Etoposide and Cisplatin regimens for 6 cycles. Ultrasound evaluation showed the appearance of a new mass, but tumor markers Alpha fetoprotein and $\beta$ hCG were normal. The patient underwent mass resection and was found to be in accordance with the condition of Growing Teratoma Syndrome.

Discussion: New mass presence in a patient with a history of immature teratoma was a condition that must be evaluated. The hypothesis GTS can occur because chemotherapy treatment can only destroy malignant cells but cannot destroy benign cells. Treatment of GTS is multi approach disciplinary, including chemotherapy and surgery.

Conclusion: Immature teratoma can leave residual disease which will later become a rare complication, namely growing teratoma syndrome. Treatment of GTS is represented by a multi- disciplinary approach, which includes chemotherapy and surgery.

\section{Introduction}

Germ cell tumors are widely considered to be morphologically and immunophenotypically homologous to germ neoplasms arising in the gonads and extra-gonads. Classification of germ cell tumors based on the World Health Organization (WHO) was divided into germinoma (resembling seminoma of the testis and dysgerminomas the ovaries), teratoma (mature, immature, and secondary malignancies), tumor yolk sac, embryonal carcinoma, choriocarcinoma, and tumor mix [1]. Germ cell malignancies account for $10 \%$ of all non-epithelial malignancies of the ovary. The most common types of germinal malignancies include dysgerminoma and 
immature teratoma. 99\% of teratoma in ovary was benign and the others was malignant. Histological analysis of immature teratomas showed that they consist not only of an immature component but also a mature component, the latter containing most of the immature neural tissue. Immature teratomas are classified into three grades due to the relative amount of immature neural tissue. Treatment were surgery and chemotherapy. This malignancy has a fairly good cure rate among ovarian malignancies [2].

Growing teratoma syndrome (GTS) is a rare complication between patients with germinal ovarian cell tumors. The incidence of growing teratoma syndrome occurs in $1.9-7.4 \%$ in all immature teratomas, characterized by an enlarged metastatic mass during adequate systemic chemotherapy and normal serum markers. Retro-peritoneal residual mass is a common finding after chemotherapy. It may contain mature teratomas, fibrotic tissue, or tumors. Mature teratomas, which unresponse to chemotherapy, may result from the evolution of malignant lesions after chemotherapy or may represent metastases from mature teratoma foci [3].

\section{Case Illustration}

An 18-year-old woman from Acehnese ethnicity, unmarried, came for routine check-ups to the Gynecological Oncology Polyclinic at Dr. Zainoel Abidin Hospital, Banda Aceh with complaint of abdominal pain that was felt occasionally. Other complaints such as bleeding from vaginal, nausea and vomiting were denied. The urination and defecation were still within normal limits. History of menarche at the age of 12 years, regular menstruation, 7 days of menstruation, changing sanitary napkins 3-4 times a day (before illness), and denied dysmenorrhea. Currently, the patient has no complaints during menstruation. The patient has undergone unilateral salpingo-oophorectomy due to ovarian tumor indications in 2019 with histopathological results of immature teratoma. After that, the patient underwent chemotherapy with a regimen of bleomycin, etoposide and cisplatin for 6 cycles in the same year. After chemotherapy, patients routinely underwent ultrasound evaluation and tumor markers. In the evaluation, there were no abnormalities in the ultrasound findings and tumor markers.

At this time, the patient claimed to feel a lump in the lower abdomen. Examination of vital signs were within normal limits. On physical examination found a flexible abdomen, intestinal peristalsis was found within normal limits and did not increase a palpable mass at the level of the symphysis pubis, mobile, without tenderness. Supportive examinations were carried out in the form of transabdominal gynecological ultrasonography, alpha fetoprotein (AFP) and $\beta$ hCG values. The ultrasound showed an anteflexed uterus measuring $7.9 \times 2.73 \times 3.77 \mathrm{~cm}$, endometrial line (+) measuring $5.42 \mathrm{~mm}$, showing a hypo hyperechoic picture with a size of $6.24 \times 4.48 \times 4.61 \mathrm{~cm}$, the origin of the mass is difficult to assess, no ascites is found, the impression is in the form of intraabdominal mass (Figure 1). AFP value is $1.82 \mathrm{ng} / \mathrm{ml}$. Furthermore, upon the finding of a growing mass, the patient was suspected of having a new intra-abdominal mass and planned a second look laparotomy.

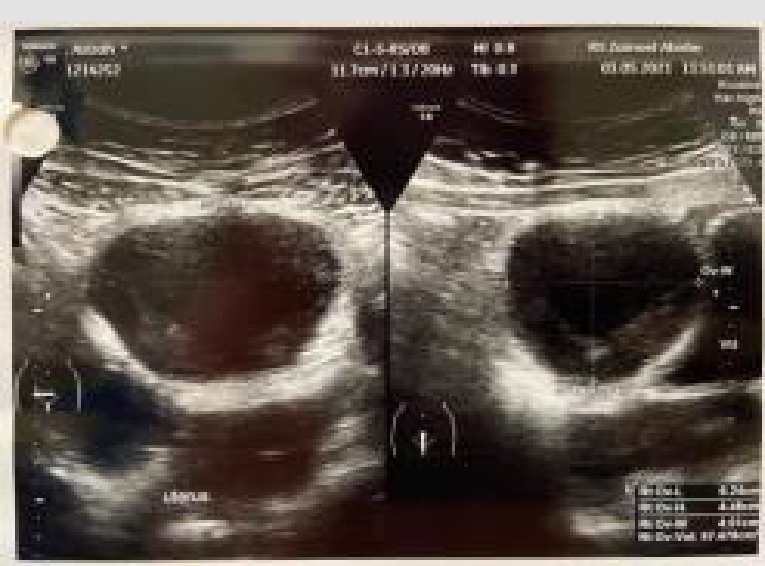

Figure 1: Mass Ultrasonography Results.

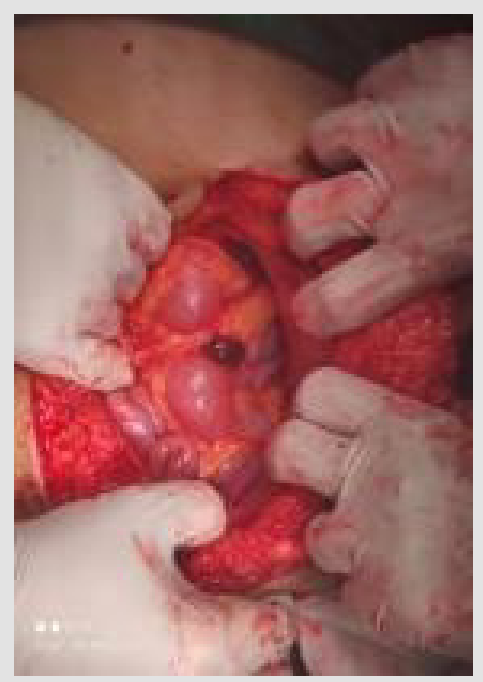

Figure 2: Intraoperative findings.

On exploration, we found the uterine and right ovary were within normal limit. It also was found 3 nodules on the peritoneum and omentum that conglomerated in the superior uterine, with the largest being $3 \times 4 \mathrm{~cm}$ and the smallest being $2 \times 2 \mathrm{~cm}$ (Figure 2 ), then adhesiolysis was performed and it was decided to take a mass and omentectomy for histopathological examination. Further exploration found nodules in the intestine measuring $2 \times 2 \mathrm{~cm}$, then a mass was taken for histopathological examination (Figure 3). Further exploration revealed no nodules in the liver. After the procedure, the patient underwent 2 days of treatment and went home after the second day of treatment. Histopathological results 
after surgery showed the results of glandular structures lined with cuboidal epithelial cells with cell nucleus morphology within normal limit. In the histopathological preparations, there were no signs leading to malignancy and no signs leading back to immature teratoma.

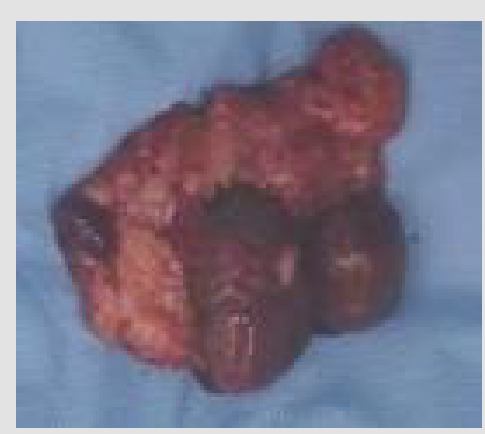

Figure 3:

(a) Nodules on the peritoneum and omentum;

(b) Nodules in the intestines.

\section{Discussion}

Immature teratoma is a type of non-epithelial malignancy in the ovary. The degree of malignancy depends on the appearance of the neuroepithelium in the tumor. The incidence of this immature teratoma is about $10-20 \%$ among germinal malignancies of the ovary. Age up to 20 years is the largest population with this disease [4]. Immature teratomas occur generally in the first 2 decades of life and are mostly absent at menopause. This tumor was determined histologically based on the grade number and degree of immaturity of the cells [5]. The patient was first diagnosed with an immature teratoma at the age of 16 years with complaints of a palpable mass in his abdomen. Then the patient was decided to undergo a surgical procedure and the histopathological results of an immature teratoma were obtained. The growth of immature teratomas is quite fast with a median time between onset of symptoms and diagnosis of 5-12 weeks. The patients were present with complain of palpable pelvic mass, and abdominal pain. Experiences of other patients with abnormal vaginal bleeding were found $12 \%$ patients presented with abdominal pain, which may be associated with capsule rupture or twisting [6,7]. Immature teratomas consist of all germ cell networks, which is endoderm, mesoderm, and ectoderm and assessed histologically. The five-year survival rate of immature teratomas is around $90 \%$ in stage I and II but it descent to $82 \%$ for stage III and $72 \%$ for stage IV. High grade histology significantly get worsens the prognosis [8].

Incidence of immature teratoma on both ovaries at the same time is very rare. These tumors can have other implants in the surrounding tissue such as the peritoneum which is usually found intraoperatively. Prognosis is depending on the histological grade of the tumor and metastatic implants [9]. Peritoneal dissemination on immature teratoma mainly on the age of the children is a rare case and often provide a poor prognosis[10]. In the pediatric population with immature teratoma, elevated serum AFP levels were found in $50 \%$ of cases, but in adult there was found in one-third of cases. Levels of cancer antigen-125 (CA-125) were also found to have a less significant increase. Immature ovarian teratoma was not associated with elevated levels of human chorionic gonadotropin (hCG). Ultrasound may or may not describe the diagnosis of this disease, depending on the number of solid or immature tissue components [6].

In pre-menopausal patients in whom the tumor usually appears on one ovary, unilateral oophorectomy and limited grade surgery should be performed. Resection of other tumors that may cause morbidity and making delay for chemotherapy should not be performed. Resection of residual mass should be happened after completion of chemotherapy [4]. Patients with early-stage disease such as stage IA have a better prognosis, and adjuvant therapy after surgery is not required. In patients with high-grade, stage $1 \mathrm{~A}$ immature teratoma, adjuvant chemotherapy is generally given, although this is sometimes questioned [4]. The commonly used combination chemotherapy regimen in the past was VAC (vincristin, actinomycin, and cyclophosphamide), however a study by the Gynecologic Oncology Group (GOG) reported that the relapse free rate in patients with incompletely resected disease was only $75 \%$. Over past 20 years have incorporated cisplatin into the treatment of these disease, and mostly with VBP (vinblastine, bleomycin, and cisplatin) in the past and now with BEP (bleomycin, etoposide, and cisplatin) [4]. GOG has evaluated of three cycle BEP therapy in fully resected stage I, II, and III ovarian germ cell tumors patients. 91 of the 93 patients $(97.8 \%)$ with non dysgerminomatous tumors were clinically disease free [4]. Administration of cisplatin can reduce the components of immature cells in teratoma derived from embryonic stem cells and pluripotent stem cells which are believed to cause apoptosis and also induce differentiation [2].

The second look laparotomy for ovarian germ cell tumor is not indicated for patients who have received adjuvant chemotherapy (eg, stages 1A, 2, and 3). However, this procedure is considered in suspected metastatic residual immature teratoma because it can lead to a rare complication such growing teratoma syndrome [4]. GTS was defined by Logothetis et al. in 1982 as a growth of benign tumor phenomenon, after removal of the primary malignant tumor during or after chemotherapy [11]. The incidence of GTS in testicular nonseminomatous germ cell is $1.9 \%$ to $7.6 \%$, and occure in $12 \%$ of ovarian germ cell tumors $[12,13]$. GTS was determined based on the following criteria: 
1. Continued growth of extra-gonadal tumor foci after diagnosis of immature teratoma or mixed germ cell tumor containing immature teratoma during or after chemotherapy with.

2. Serum markers (AFP and hCG) previously increased become normal and.

3. Histopathology shows mature teratoma in the resected tumors. 6 Complete resection is important for the treatment of GTS. The presence of residual mass after surgery is a risk factor for recurrence GTS $[14,15]$.

Patients who have undergone a laparotomy have a mass taken in the omentum and intestine. The histopathological results that we found did not find any malignancy in the nodule preparation. Preoperative findings with the presence of a mass after chemotherapy and a normal level of tumor marker AFP in the patient, two of the three GTS criteria proposed by Logotheis were met. The third condition that is met is the presence of a mature teratoma mass in a new mass histopathological preparation. In terms of histopathological results, this patient had histopathological results that did not show malignancy in the preparation. Treatment of GTS is multi-disciplinary approach, such as chemotherapy and surgery. GTS is diagnosed during or after the completion of chemotherapy. In case when GTS is recognized during chemotherapy, completion the early chemotherapy cycle is highly recommended. Discontinuation of chemotherapy is warranted only in the presence of vital symptoms caused by an increase in tumor size and in the presence of compression in surrounding organs (eg, intestine, lung, liver) with life- threatening organ failure. Only in this case, early resection of mass is acceptable. Improvements in oncological outcomes were achieved by following three steps:

1. Early recognition growing tumor size and elevated tumor markers during chemotherapy,

2. Completion of a number of early stages of the chemotherapy course under thorough monitoring of clinical size and appearance, and

3. Complete resection of growing mass $[16,17]$.

According to Hamayun Imran et al. in his study stated that majority of GTS case have been reported in adults after adjuvant chemotherapy for all women except grade 1 tumor stage IA [18]. In a study conducted by Julie My Van Nguyen et al., stated that currently the incidence of GTS after adjuvant chemotherapy is becoming more common, as evidenced by the fact that there were 15 samples receiving adjuvant chemotherapy, 6 of whom had GTS so that further examination or required. The follow up was periodic patients with a history of immature teratoma through examination of tumor markers and imaging [19]. In a study conducted by Song
Li et al., stated that GTS has a very good prognosis. Patients with GTS were found to be disease-free for 40.3 months (range 1-216 months, $\mathrm{n}=48$ ) based on the median follow-up score. In addition, regular imaging and serum tumor markers follow-up are important [15]. GTS can develop in children, and the tumor size are same between adolescents and adults. Furthermore, GTS progression from primary germ cell tumors may be more rapid in children than adolescents and adults. Complete resection of all GTS tissue is recommended, although fertility sparing surgery should be considered whenever possible [20].

\section{Conclusion}

Immature teratoma can leave residual disease which will later become a rare complication, namely growing teratoma syndrome. Treatments of GTS are multi- disciplinary approach such as chemotherapy and surgery.

\section{Conflict of Interest}

No conflict of interest with any institution/organization.

\section{References}

1. Hattab EM (2018) 18 - Germ Cell Tumors. Second Eddie. Practical Surgical Neuropathology: A Diagnostic Approach A Volume in the Pattern Recognition Series. Elsevier Inc., pp. 423-442.

2. Kurata A, Takanashi M, Ohno Sichiro, Fujita K, Kuroda M (2021) Cisplatin induces differentiation in teratomas derived from pluripotent stem cells. Regenerative Therapy 18: 117-126.

3. Boukettaya W, Hochlaf M, Boudagga Z, Ezzairi F, Chabchoub I, et al. (2014) Growing Teratoma Syndrome After Treatment of a Nonseminomatous Germ Cell Tumor: A Case Report and a Review of Literature. Urology Case Reports 2(1): 1-3.

4. Berek JS, Friedlander ML, Hacker NF (2015) Germ Cell and Nonepithelial Ovarian Cancer. In: Berek \& Hacker's Gynecologic Oncology ( $6^{\text {th }}$ Edn.)., Philadelphia: Wolters Kluwer, pp. 530.

5. Penick ER, Hamilton CA, Maxwell GL, Marcus CS (2018) Germ cell, stromal, and other ovarian tumors. Ninth Edit. Clinical Gynecologic Oncology. Elsevier Inc., pp. 290-313.e7.

6. Medeiros F, Strickland KC (2017) Chapter 26 - Germ Cell Tumors of the Ovary ( $3^{\text {rd }}$ Edn.)., Diagnostic Gynecologic and Obstetric Pathology. Elsevier Inc., pp. 949-1010.

7. Gemmell JE (2016) Ovarian Teratoma. Journal of the Royal Society of Medicine 4: 285-288.

8. Cochrane E, Frost K, Dinobile C, Kim S, Burke W (2020) Immature teratoma diagnosed and treated during pregnancy and later complicated by growing teratoma syndrome: A case review with clinical considerations. Gynecologic Oncology Reports 32: 100566.

9. Bidus MA, Elkas JC, Rose GS (2012) Germ Cell, Stromal, and Other Ovarian Tumors ( $8^{\text {th }}$ Edn.).,. Clinical Gynecologic Oncology: Elsevier Inc., pp. 329-356.

10. Brind'Amour A, De Guerke L, Fortin S, Gervais MK, Dubé P, et al. (2020) Recurrent High-grade Ovarian Immature Teratoma with Peritoneal Dissemination. Journal of Pediatric and Adolescent Gynecology 33(5): 586-589. 
11. Logothetis C, Samuels M, Trindale A (1982) The Growing Teratoma Syndrome. Cancer 50: 1629-1635.

12. Zagame L, Pautier P, Duvillard P (2006) Growing teratoma syndrome after ovarian germ cell tumors. Obstetrics and Gynecology 108: 509-514.

13. Gorbatiy V, Spiess P, Pisters L (2009) The growing teratoma syndrome: current review of the literature. Indian Journal of Urology 96: 784-791.

14. Wang D, Zhu S, Jia C, Cao D, Wu M, etal. (2020) Diagnosis and management of growing teratoma syndrome after ovarian immature teratoma: A single center experience. Gynecological Oncology 157(1): 94-100.

15. Li S, Liu Z, Dong C, Long F, Liu Q, et al. (2016) Growing teratoma syndrome secondary to ovarian giant immature teratoma in an adolescent girl a case report and literature review. Medicine (United States) 95(7): e2674.

16. Hiester A, Nettersheim D, Nini A, Lusch A, Albers P (2019) Management, Treatment, and Molecular Background of the Growing Teratoma Syndrome. Urological Clinics of North America 46(3): 419-427.

ISSN: 2574-1241

DOI: 10.26717/BJSTR.2022.41.006615

Hasanuddin. Biomed J Sci \& Tech Res

(C) (P) This work is licensed under Creative

Submission Link: https://biomedres.us/submit-manuscript.php
17. Paffenholz P, Pfister D, Matveev V, Heidenreich A (2018) Diagnosis and management of the growing teratoma syndrome: A single-center experience and review of the literature. Urological Oncology: Seminars and Original Investigations 36(12): 529.e23-529.e30.

18. Imran H, Siddiqui AH, Wilson F, Pashankar F (2020) Growing Teratoma Syndrome after Chemotherapy for Ovarian Immature Teratoma. Journal of Pediatric Hematology/Oncology 42(7): e630-633.

19. Van Nguyen JM, Bouchard-Fortier G, Ferguson SE, Covens A (2016) How common is the growing teratoma syndrome in patients with ovarian immature teratoma? International Journal of Gynecological Cancer 26(7): 1201-1206.

20. Oyama T, Noda T, Washio K, Shimada A (2020) Pediatric growing teratoma syndrome of the ovary: A case report and review of the literature. Medicine 99(38): e22297.

\begin{tabular}{|l} 
BIOMEDICAL \\
RESEARCHES
\end{tabular}$\quad \begin{aligned} & \text { Assets of Publishing with us } \\
& \text { - Global archiving of articles }\end{aligned}$

